\title{
Updating Monte Carlo collision generators with LHC data up to $\sqrt{s}=8 \mathrm{TeV}$
}

\author{
Jean-Noël Capdevielle ${ }^{\mathrm{a}}$ \\ APC Université Paris Diderot, 10 rue A. Domon et V. Duquet, 75013 Paris and Académie des Sciences d'Outre Mer, \\ 15 rue La Pérouse, 75116 Paris, France
}

\begin{abstract}
Monte Carlo collision generators describing the multiple production of secondaries in very high energy interactions can be updated taking into account the LHC data available now up to $\sqrt{s}=8 \mathrm{TeV}\left(3.2 \cdot 10^{16} \mathrm{eV}\right)$. The energy range of the so-called knee energy region in cosmic ray physics $\left(2 \cdot 10^{15}-10^{17} \mathrm{eV}\right)$ for proton primaries will soon be completely covered by the LHC data. An extension of the model HDPM of CORSIKA has been developed on a base of the recent measurements by LHCb, CMS, TOTEM... The new model GHOST involving a 4-source production is presented here. It correctly reproduces the pseudo-rapidity distributions of charged secondaries and can help the approach of the data in the mid and forward rapidity region, especially in the complex case of TOTEM. In parallel, simulations of cascades and EAS are also carried out in order to understand unexplained results in energy distributions of very high energy $\gamma$ 's.
\end{abstract}

\section{Introduction}

More than 3 decades ago, the CERN S $p \bar{p} \mathrm{~S}$ data provided important results in the energy range $\sqrt{s}=200-$ $900 \mathrm{GeV}$ implemented rapidly in cosmic ray simulation, especially with the potential of UA5, UA4, UA2, UA1 measurements [1]. This energy range was extended near five years later when the CDF detector started operating at Fermi-lab up to $\sqrt{s}=1.8 \mathrm{TeV}$ equivalent to an energy exceeding $1.6 \cdot 10^{15} \mathrm{eV}$ close to the cosmic ray knee at $3 \cdot 10^{15} \mathrm{eV}$ [2].

Measurements of the charged particles density of pseudorapidity, $\eta$, were among the earliest observations made at $\mathrm{S} p \bar{p} \mathrm{~S}$, together with the average central pseudorapidity density $\rho(0)$. Simultaneously, single diffractive, double diffractive cross sections were estimated as well as the transverse momentum, $p_{t}$, distribution of charged secondaries. Fermi-lab provided consistent results with the CERN measurements, allowing a knowledge of basic features of high energy cosmic ray interactions which were previously established by extrapolations from ISR data. Those circumstances have generated more reliable simulations of EAS exceeding $10^{15} \mathrm{eV}$, especially for electron and muon lateral distributions, important to estimate the primary energy.

Following the earliest run of the LHC, measurements of the density of pseudo rapidity of charged secondaries have been performed by ALICE and CMS at $\sqrt{s}=0.9,2.36 \mathrm{TeV}$. The data is consistent with the results of UA5 at $\sqrt{s}=0.9 \mathrm{TeV}$ and there are very small discrepancies between proton-proton collisions and proton-antiproton collisions. Up to $\sqrt{s}=2.36 \mathrm{TeV}$, all the densities are easily reproduced with a small incremental tuning of existing models. However, this data concerns mainly the central pseudo rapidity region with $\eta<3$. Recently, new measurements have been achieved at $\sqrt{s}=$

a e-mail: capdev@apc.univ-paris7.fr
$7-8 \mathrm{TeV}$ by LHCb, CMS and TOTEM: the densities were collected up to $\eta<7$ providing more complete information in the mid-rapidity region and allowing the comparison of the pseudorapidity profiles observed and simulated over a large band of rapidity.

In the sections that follow, we shall discuss the new improvements in cosmic simulation suggested by the most recent results of the LHC, especially the data of ATLAS, CMS, TOTEM, LHCb... up to $\sqrt{s}=8 \mathrm{TeV}$ used as the largest energy available to elaborate and testify the new Monte Carlo generator suitable for cosmic ray simulations.

Section 2 is devoted to the recent tendencies observed from $\mathrm{S} p \bar{p} \mathrm{~S}$ to $\mathrm{LHC}$ in important features of high energy interactions.

Section 3 is focused on the four component model GHOST (Generator Hadronic Oversteering Secondary Treatment) describing the transition from the original HDPM (Hybrid Dual Parton Model) suggested by the transition from a double pomeron structure to a multiple pomeron structure.

Section 4 evocates some measurements not explained in cosmic ray data above $\sqrt{s}=5 \mathrm{TeV}$, especially in the case of high energy spectra of secondaries measured in EAS and Gamma-ray families.

Finally in Sect. 5, we outline the possible consequences of unexpected changes in models of interaction above $\sqrt{s}=5 \mathrm{TeV}$ for extrapolations at higher energies and interpretation of cosmic ray data at ultra high energy.

\section{New tendencies in multiple Production from $S p \bar{p} S$ to $\mathrm{LHC}$ energies}

Each new step in energy reached by the colliders is an opportunity to evaluate the predictions of the models. The simulation of the hadronic cascade in cosmic ray physics needs at least the parameters describing the different cross sections, the total multiplicity of secondaries, their

This is an Open Access article distributed under the terms of the Creative Commons Attribution License 4.0, which permits unrestricted use, distribution, and reproduction in any medium, provided the original work is properly cited. 
energy and transverse momenta distributions, the energy dependence of those parameters and the statistic laws governing their fluctuations. The violation of Koba, Nielsen, Olesen (KNO) scaling observed in UA5, for instance, introduced the negative binomial distribution well adapted to the prediction of multiplicities of secondaries in cosmic ray cascades.

\subsection{Microscopic and statistical models}

Cosmic ray high energy interactions are mainly understood with the "soft physics" data provided by the colliders. Therefore, the approach of microscopic models starts from the parton distribution functions, distinguished for valence quarks and diquarks, sea quarks and gluons. In the case of $p$-p collisions, there are common features between models used in accelerators or in cosmic ray simulations, respectively models such as the Dual Parton Model, Phojet, Pythia on one side, models like QGSJet, Sybill on the other side [3].

The geometrical and statistical models such as the cluster model GENCL of UA5 [4] are more connected with the phenomenological features of the experimental data and built on analogy with quantum optics. Similarly, the Hybrid Dual Parton Model (HDPM) has been inspired by the most simple double pomeron structure of DPM: it represents the pair of quark-diquark chains stretched between the valence quark and diquark of the projectile and the target by one pair of gaussian functions with a correct overlap to reproduce the expected rapidity distribution with the profile of one plateau with gaussianshaped wings [3]. Like in the International Court of Justice of the Hague, the recent results of very energetic colliders select the most reliable models and dictate further improvements.

Unfortunately, in the interpretation of cosmic ray data, the approach has a limit due to the difficulties of measurement in the very forward region of rapidity and the determination of the properties of the leading cluster. In other words, the role of the pilot particle fundamental in hadronic cosmic ray cascades remains dependent on adequate assumptions.

All the models have been extended to $\mathrm{p}-\mathrm{A}$ and $\mathrm{A}$ A collisions in order to describe the collision of cosmic ray hadrons with air constituents and the comparison with LHC data becomes also possible. Conversely, there exist also models elaborated directly for collisions of heavy nuclei which can be tuned to describe $p$-p collisions; this is the case, for instance, of the non-equilibrium statistical relativistic diffusion model (RDM) based on a FokkerPlanck type transport equation [5]. Hence, the earliest and most simple data produced by colliders and accelerators is the charged Non Single Diffraction (NSD) distribution. The characteristics of this distribution: average central rapidity density $\rho(0)$, total average multiplicity of charged secondaries $n_{c h}$ (as far as it can be approached by the integration of the densities collected on up to the smallest angles of emission), the maximum and the full width at half maximum (FWHM), transverse momentum distribution, are the normal tests of the preliminary validity of the models recognized.

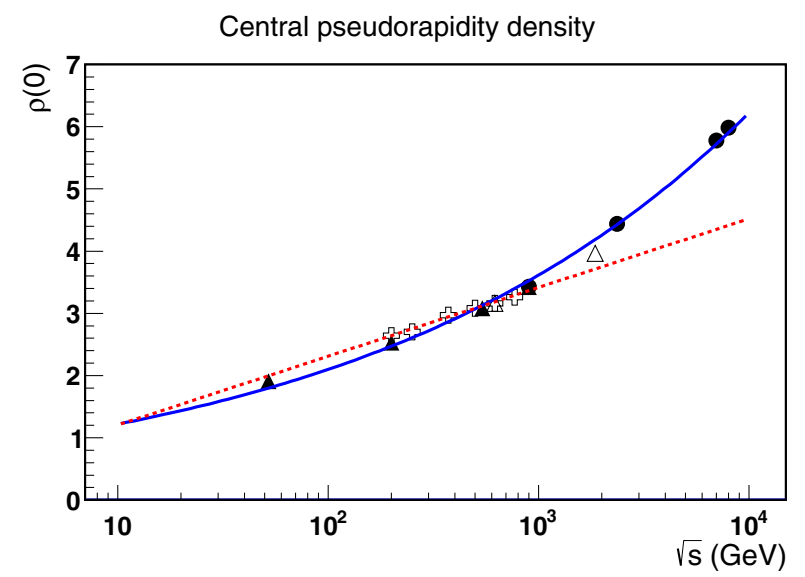

Figure 1. Central pseudorapidity density dependence on $\mathrm{s}$ for $E_{0}=10^{12}-10^{17} \mathrm{eV}$. Under the power law adjustment, the original extrapolation of UA5 (dashed) is superposed. Experimental points are from ISR and UA5 $(\bullet), \operatorname{UA} 4(+), \operatorname{CDF}(\triangle)$, following the compilation of CMS-TOTEM $(\bullet)$.

A next step of quality is reached with additive criteria adapted to the same pseudorapidity distribution data considered in small bands of multiplicity i.e. in a semiinclusive analysis.

\subsection{Central pseudo-rapidity densities at LHC}

The dependence $\rho(0)$ on $\sqrt{s}$ exhibits an unexpected enhancement at the most recent energies attained by the LHC [6]. Therefore, we have first fitted the energy dependence of central pseudorapidity densities (here for NSD, only) $\rho(0)$ and perform a numerical extrapolation as follows in the energy range $E_{0}=10^{12}-10^{17} \mathrm{eV}$ :

$$
\begin{gathered}
\rho(0)=0.708 s^{0.118} \\
\rho(0)=\rho_{1}(0)=0.24 \ln (s)+0.1(\sqrt{s} \leq 1.292 \mathrm{TeV}) \\
\rho(0)=\rho_{2}(0)=0.666 \ln (s)-6(\sqrt{s} \geq 1.292 \mathrm{TeV})
\end{gathered}
$$

The rise of density can be adjusted correctly with either a power law dependence in Fig. 1 or within a linear dependence by one pair of $\ln (s)$ functions in Fig. 2, $\rho_{1}(0)$ and $\rho_{2}(0)$. As it appears in Fig. 2, the $\ln (\mathrm{s})$ description above $\sqrt{s} \geq 1.292 \mathrm{TeV}$ might correspond to the addition of a central contribution in the knee energy region of cosmic rays $\rho_{3}(0)=0.426 \ln (s)-6.1$ as $\rho_{2}(0)=\rho_{1}(0)+$ $\rho_{3}(0)$.

\subsection{Normalized pseudorapidity density and semi inclusive data}

An interesting property of the normalized central density of pseudorapidity has been observed also in the semi inclusive data in colliders and accelerators [7]. A new kind of empirical scaling has been pointed out in the relation $\rho_{n}(0) / \rho(0)=f(z)$ instead of $f(z, s)$, i.e. independent from $\sqrt{s}$ where $\rho_{n}(0)$ is the central density for a given charged multiplicity $n_{n}, \rho(0)$ the average central 


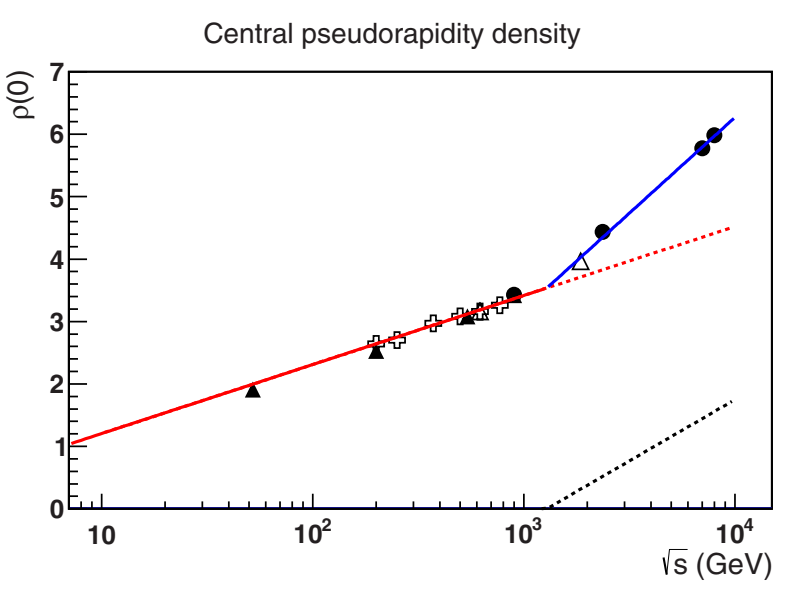

Figure 2. Central pseudorapidity density $\ln (\mathrm{s})$ dependence on $E_{0}=10^{12}-10^{17} \mathrm{eV}$. At bottom (dashed) additive contribution above $\sqrt{s} \geq 1.292 \mathrm{TeV}$.

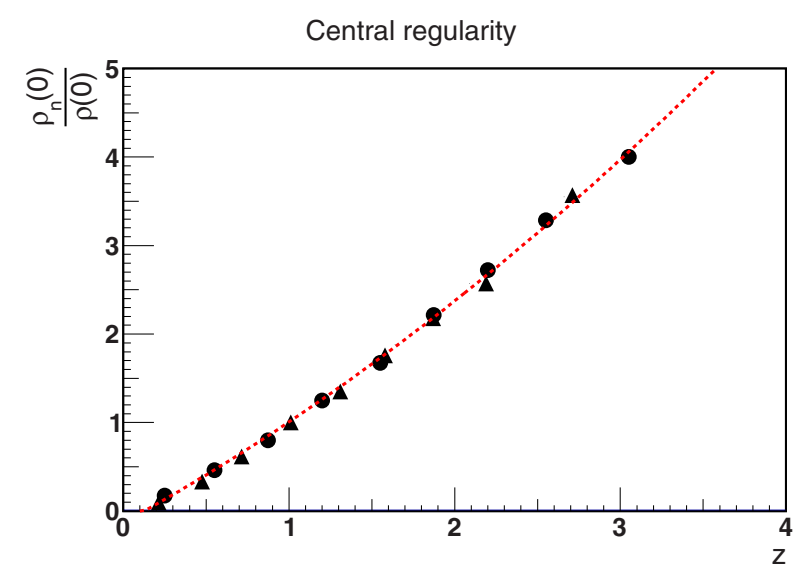

Figure 3. Empirical scaling function $\zeta=\mathrm{f}(\mathrm{z})$ for $\sqrt{s}=200$ $($ dark $\triangle$ ), 546 and $900 \mathrm{GeV}(\bullet)$ for NSD collisions.

density. The KNO variable $z=n_{n} / n_{c h}$ is the normalized multiplicity. The general dependence on $\mathrm{z}$ is shown in Fig. 3. Following our adjustment, we propose for $f(z)$ the numerical representation:

$$
\zeta=f(z)=0.11499 z^{2}+1.0231 z-0.1319 .
$$

This new kind of scaling is not yet confirmed in LHC data as the semi-inclusive measurements are performed only in small windows of pseudorapidity; nevertheless, the important fluctuations of those reduced distributions suggest a similar amplitude of the KNO violation.

\section{NSD profile and models at $\sqrt{s}=$ 7-8 TeV}

Taking into account the data recorded by several experiments in LHC, Atlas, CMS, TOTEM, LHCb [8], it becomes possible to obtain the pseudorapidity profiles of the NSD distributions of charged secondaries in central and mid-rapidity regions close to the fragmentation region, thanks to the case of TOTEM and LHCb extending the measurements up to 7 units of pseudorapidity. It happens that the data of TOTEM and LHCb doesn't agree with the

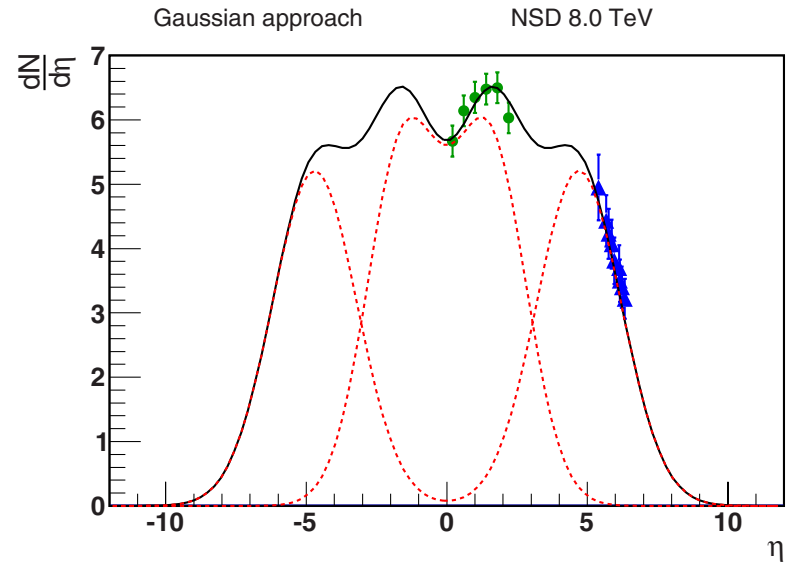

Figure 4. NSD data at $\sqrt{s}=8 \mathrm{TeV}$ [6] described by 4 gaussians functions.

prediction of the models, whereas the central densities still correspond to the expectation from models used so far.

Several questions are generated by the measurements performed by TOTEM and LHCb concerning the data analysis and the selection of the NSD events. The systematical and statistical uncertainty of density measurements is reported by TOTEM as $6-8 \%$. The situation looks similar for the inelastic component which is not considered in this paper. We underline here that the corrections and systematics between CMS and TOTEM are stll in progress.

Just fitting the energy behaviour of the FWHM $\sigma_{H W}$, we obtained the energy $\ln (\mathrm{s})$ growth in NSD distribution

$$
\sigma_{H W}=0.382 \ln (\sqrt{s})+0.5
$$

up to $8 \mathrm{TeV}$, larger than the extrapolations suggested by the behaviour observed when going from $\sqrt{s}=53 \mathrm{GeV}$ up to $\sqrt{s}=900 \mathrm{GeV}$.

\subsection{Gaussian and hyperbolic approaches}

A large number of models testified up to Fermilab maximal energy $\sqrt{s}=1.8 \mathrm{TeV}$ have reproduced very well the pseudo-rapidity densities measured in the LHC up to $\sqrt{s}=2.2 \mathrm{TeV}$ in the central rapidity region. More recently, especially after densities observed in the mid-rapidity region, difficulties appeared at the most important energies reached $\sqrt{s}=7-8 \mathrm{TeV}$. The preliminary topological approach of the profile of the NSD distributions of CMS and TOTEM at $\sqrt{s}=8 \mathrm{TeV}$ is shown in Fig. 4 assuming the superposition of 4 gaussian functions symmetric in the CMS frame. Only the distributions of rapidity of high energy secondaries are aproximately similar to the pseudo-rapidity distributions for the region of y much greater than 0 . Therefore, the gaussian adjustment in the forward region centered at $\eta= \pm 4.7$ with a width 1.5 (amplitude 5.21) suggests the profile of the rapidity distribution above 4 units of pseudorapidity. The gaussian functions centered at $\eta=$ \pm 1.53 with a width 1.3 (amplitude 5.6) remains different from the actual behaviour of the rapidity distribution, but altogether the profile of one plateau with gaussian wings looks unlikely. Another approach can also be 
Hyperbolic approach NSD 8.0 TeV

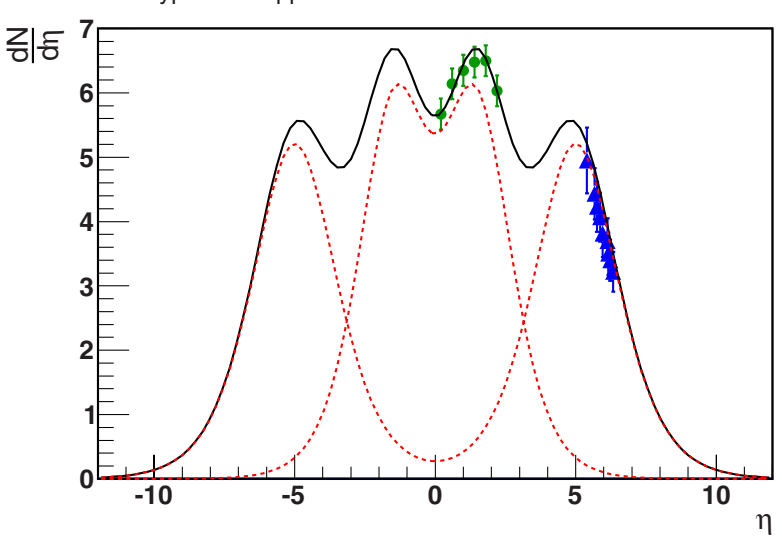

Figure 5. NSD data at $\sqrt{s}=8 \mathrm{TeV}$ [6] described by 4 hyperbolic cosine functions.

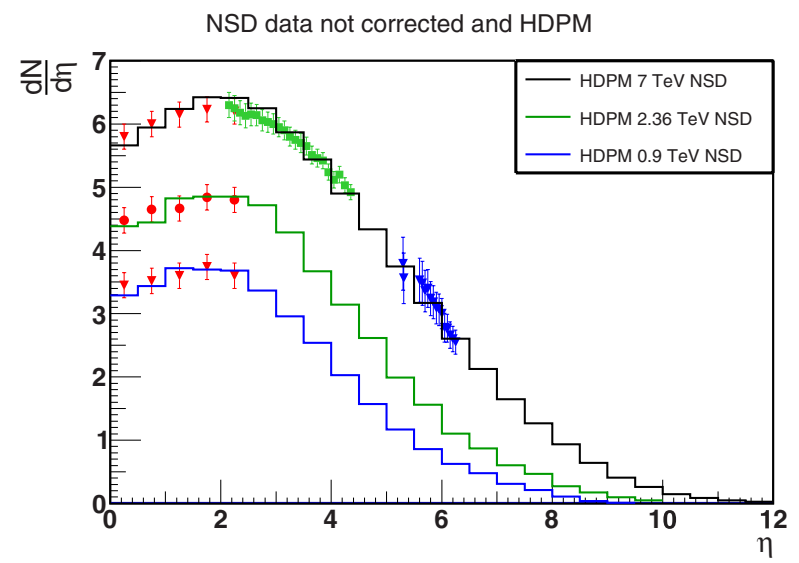

Figure 6. Preliminary NSD data at $\sqrt{s}=7 \mathrm{TeV}$ and HDPM histogram simulated. Experimental points from left correspond to CMS, LHCb and Totem measurements, compilated in [6] and reference herein.

carried with 4 hyperbolic cosine functions in Fig. 5. This pure topological exercise helps the earliest steps of the multi sources Monte Carlo generation from the genuine rapidity distribution; it gives the opportunity of the close behaviour between pseudorapidity and rapidity distributions at mid and forward rapidity region. In fact the assumed "genuine" rapidity distribution is optimized by successive approximations taking into account in addition the previous energy guide lines of HDPM and the scaling function $\zeta=f(z)$.

A first attempt to reproduce the first NSD distribution presented with HDPM [9] as inserted in CORSIKA [10] hereafter reflects an apparent agreement with CMS and TOTEM data at $\sqrt{s}=7 \mathrm{TeV}$ as shown in Fig. 6 .

Observing that the data presented here for TOTEM were inelastic, whereas the data for CMS were NSD, we reproduce in Fig. 7 a possible comparison with NSD data enhancing the inelastic densities of TOTEM [5] following the ratio NSD/inelastic in UA5. It appears clearly that HDPM cannot reproduce such a NSD profile in Fig. 7.

\subsection{4 sources GHOST model}

A model with 4 sources is probably more suitable than HDPM to take into account the multi-pomeron structure
NSD data corrected and HDPM

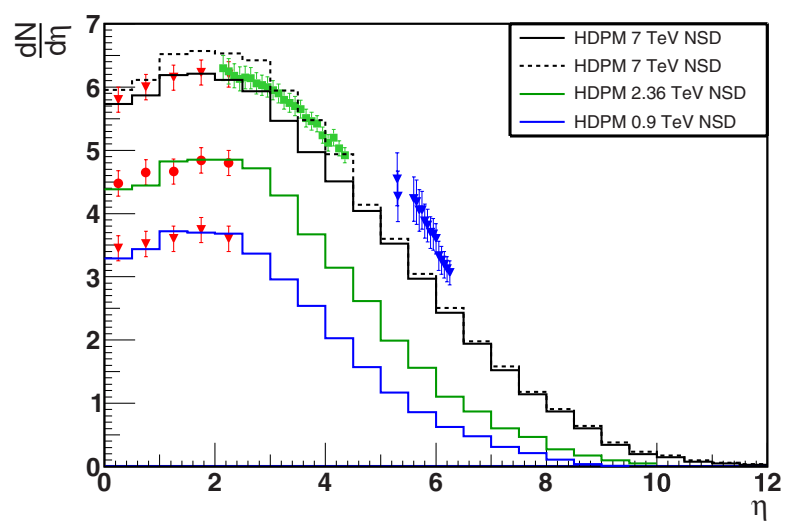

Figure 7. Preliminary NSD data at $\sqrt{s}=7 \mathrm{TeV}$ where TOTEM possible NSD densities of pseudorapidity are roughly assumed after converting the inelastic distribution measured (for $\eta \geq 5.3$ ) and HDPM NSD histogram.

promoted in microscopic physics. The extension from HDPM is carried as follows:

- $p_{t}$ distribution conserved from HDPM

- Rapidity distribution with 4 gaussian functions

- Fluctuation of total multiplicity with the negative binomial ditribution as in UA5 and in HDPM

- Composition of secondaries (pions, kaons, $\eta^{\prime} s$, baryons) conserved from HDPM

- Conservation of longitudinal and transverse momenta, conservation of total energy.

The rapidity distribution used for the Monte Carlo generation of the individual rapidities is described herafter:

$$
\begin{gathered}
\frac{d N}{d y}=\sum_{i=1}^{i=2} a_{i}\left(e^{-0.5 u_{i}}+e^{-0.5 v_{i}}\right) \\
u_{i}=\left(\frac{y-y_{i}}{\sigma_{i}}\right)^{2}, v_{i}=\left(\frac{y+y_{i}}{\sigma_{i}}\right)^{2} .
\end{gathered}
$$

The gaussian functions have been preferred in order to generate more rapidly the individual rapidities of one set of secondaries. First the negative binomial distribution is used as in HDPM to generate a given total multiplicity $n$, then $\mathrm{n}$ values $y_{i}$ are generated in parallel with $\mathrm{n} p_{t i}$ giving the individual energy [9] $E_{i}=m_{\perp} \cos h\left(y_{i}\right)$ and longitudinal momentum $p_{z i}=m_{\perp} \sin h\left(y_{i}\right)$. Here $m_{\perp}$ is the transverse mass of the particle defined by $m_{\perp}^{2}=m^{2}+p_{t}^{2}\left(m, p_{t}\right)$ are respectively the mass and the transverse momentum of the particle generated). The production of secondaries is based on the semi-inclusive data governed by the integrodifferential system:

$$
\begin{aligned}
& \frac{d N}{d y}_{y=0}=m_{r} \frac{d N}{d \eta}_{\eta=0} \\
& \int \frac{d N}{d y} d y=z<n>.
\end{aligned}
$$

In this equation system the parameter $m_{r}$ represents the ratio of central rapidity density to central pseudorapidity 
Pseudo-Rapidity Distribution for NSD at $\sqrt{S}=8 \mathrm{TeV}$

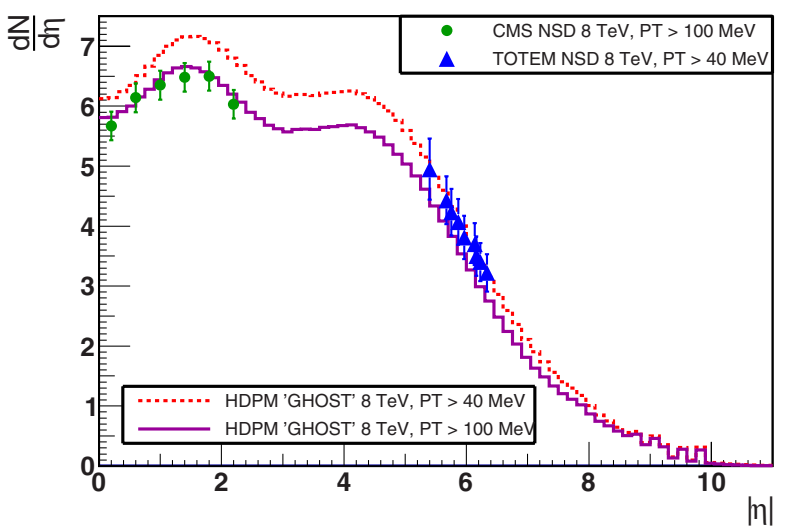

Figure 8. NSD data of CMS and TOTEM reproduced with GHOST. The main features of GHOST are here the average multiplicity $n_{c h}=85$, centers and width of gaussian generating the rapidities in central region respectively $Y_{1}= \pm 1.05$ and $\sigma_{1}=$ 0.95 , in mid-rapidity region $Y_{2}= \pm 4.1$ and $\sigma_{1}=1.8$.

density, $<\mathrm{n}>$ being the total average multiplicity. The constraints of both equations establish a link in the solutions between the center $Y_{i}$ and the width $\sigma_{i}$ of the gaussian functions describing the densities for a given multiplicity $\mathrm{n}$ selected by the negative binomial distribution.

$$
Y_{i}=\sigma_{i}\left(2 \ln \left(\frac{z<n>}{\sqrt{(2 \pi) \zeta \sigma_{i}}}\right)\right)^{0.5} .
$$

The relation (4) expressing the scaling of $\zeta=f(z)$ determines the most favourable position of the center of each gaussian and avoids a large number of rejections, $Y_{i}$ replacing the average value obtained for $z=1$. The Monte Carlo generation can produce random deviates with $50 \%$ of the particles in each function or contrasted between gaussian functions in central and mid-rapidity regions (for instance $60 \%$ against $40 \%$ ): those proportions have to be taken into account when using the conservation relations (7) and (8).

Using the 4-gaussian generation of GHOST we obtain a set of parameters in agreement with data of both CMS and TOTEM presented in Fig. 8. The histogram with dotted points corresponds to all the NSD charged secondaries, whereas the solid histogram takes into account the different conditions of the registration i.e. $p_{t} \geq 100 \mathrm{MeV}$ in CMS and $p_{t} \geq 40$ in TOTEM. Other combinations of parameters appear to be able to reproduce simultaneously the NSD measurements of CMS and TOTEM in agreement with a modest inclination of the "plateau", but they require larger multiplicities (i.e. < $n_{c h}>\geq 85$ ) not measured up to now, which could be also in agreement with the increase observed for central pseudorapidity at $7-8 \mathrm{TeV}$.

\subsection{Comparison with other models}

The same calculation as in Fig. 8 has been reproduced (Fig. 9) after implementing in CORSIKA option INTEST the generator GHOST for comparison with HDPM and QGSJET models. It appears that the general position of

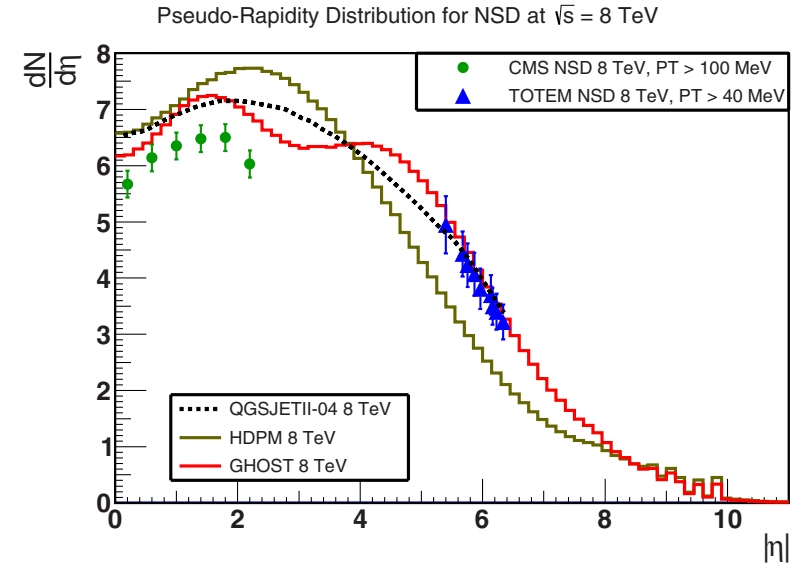

Figure 9. NSD data of CMS and TOTEM compared with models GHOST, HDPM, QGSJETII-04 following [6].

TOTEM data and the profile of the NSD distribution above 5 units of rapidity cannot be obtained by HDPM and QGSJET. In contrast, the total profile can be reproduced with the 4 sources model GHOST using different values of average multiplicity $\langle n\rangle$ and $\left.<n_{c h}\right\rangle$. In spite of success with a 3 sources model [5] in p-A collisions, the comparison was not positive for $\mathrm{p}-\mathrm{p}$ collisions, but we suppose that the parameters used by the authors have to be tuned differently. We have observed that the overlap of the gaussian distributions between central and midrapidity region plays a role in the profile of the distribution, for instance the emergence of the second maximum in the forward as well as in the backward direction when adopting the smallest multiplicities. Those peaks can also disappear if larger values are taken for the total average multiplicity $<\mathrm{n}>$.

\section{Further investigations with the inelastic component in LHC and other cosmic ray data}

The integral energy distribution of $\gamma$ 's has been measured up to $\sqrt{s}=15 \mathrm{TeV}$ in Tien Shan experiment [11]. Using a kind of Feynman's x parameter $\left(\frac{E_{\gamma}}{E_{0}}\right)$ in the laboratory frame, the authors pointed out a kind of scaling in the average integral energy distribution for primary energies $E_{0} \leq 1.05 \cdot 10^{16} \mathrm{eV}$ (i.e. $\sqrt{s} \leq 4.6 \mathrm{TeV}$ ). This scaling disappears at higher energies and the integral energy distribution becomes steeper and steeper.

The increasing steepness of this energy spectrum for $\sqrt{s} \geq 2 \mathrm{TeV}$ is not reproduced by the simulation with QGSJET model as seen in Fig. 10. The simulations of the $\gamma$ 's integral energy distributions performed with QGSJET take into account the emulsion chamber terminology ( $\gamma$ 's means photons and $\mathrm{e} \pm$ ): a general scaling is observed for all the primary energies, the profile remaining similar to the distribution shown in Fig. 10 for a primary proton with energy $E_{0}=10 \mathrm{PeV}$. It also confirms the general scaling seen under $10 \mathrm{PeV}$ in the Tien Shan experiment. The discrepancies concerning the lowest energetic $\gamma$ 's at $x \leq 10^{-3}$ can be explained as they are falling out of the limits of the chambers covering only $162 \mathrm{~m}^{2}$ and the 
Gamma Integral Energy Spectrum

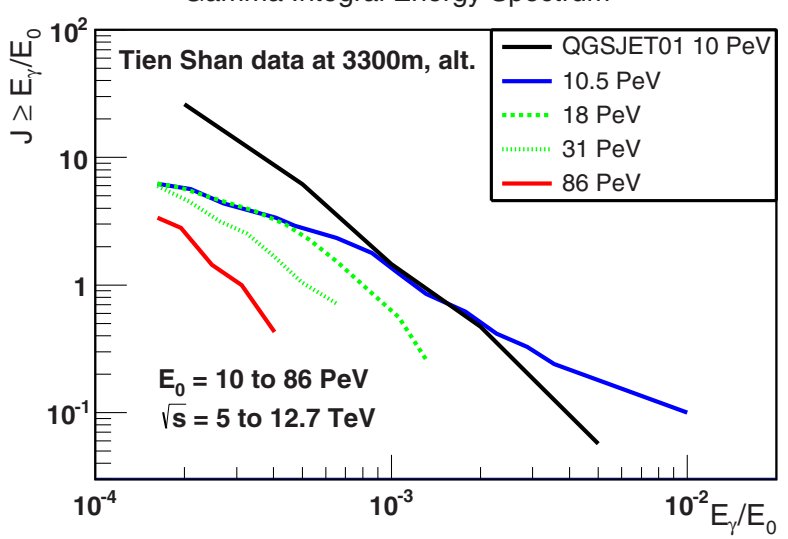

Figure 10. Very high energy $\gamma$ 's integral energy spectra and simulation with QGSJET model at the altitude of Tien Shan experiment.

Gamma Integral Energy Spectrum

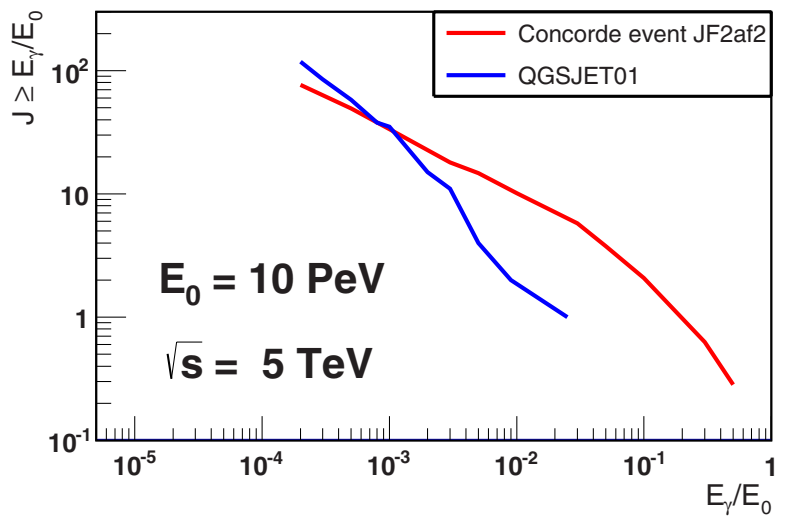

Figure 11. Very high energy $\gamma$ 's integral energy spectrum in Concorde and simulation with QGSJET model.

approximate scaling can be justified up to $E_{0} \leq 10 \mathrm{PeV}$. More extended simulations are in progress to explain the violation of scaling seen in the experimental profiles at $18,31,86 \mathrm{PeV}$. This needs integration of triggering conditions of the experimental array together with GHOST model in progress in CORSIKA.

A similar steepness appears also in one event measured on Concorde at an altitude of $17 \mathrm{~km}$ (in the most energetic collision recorded flying one emulsion chamber during 500 hours) with an energy $\sqrt{s}=5 \mathrm{TeV}$. Assuming a distance near $300 \mathrm{~m}$ above the emulsion chamber for the original collision, we note that the simulation with QGSJET herealso is unable to reproduce the integral energy spectrum as shown in Fig. 11.

Both experiments suggest that the most energetic $\gamma$ 's correlated with the leading cluster are missing. In both cases new simulations are necessary to take into account more accurately the experimental conditions, for instance in Tien Shan also for the primary energy measurement by the EAS array and the selection of the Gamma ray families contained in the emulsion chamber. In the case of Concorde more simulations are necessary to appreciate the effect of the altitude of the first collision.

\section{Conclusion}

We have shown that the 4 sources model GHOST is a very fast Monte Carlo collision generator able to reproduce the NSD data up to $\sqrt{s}=8 \mathrm{TeV}$. One pair of sources in central region has to be centered at $\pm 1.0-1.4$ units of rapidity with a width 1.2-1.4 and another pair of sources has to be centered in mid-rapidity region at $\pm 4.0-4.5$ with widths in the range 1.5-1.9 for the gaussian functions. Whenever this attempt has been concentrated on the NSD component, we note that the production of those last sources in mid-rapidity region has a profile similar to the hadronic decay of one gauge boson (probably accidental). Several combinations of parameters can explain the data collected up to now, for instance the total average multiplicity, the largest multiplicities (85-90 for charged) giving one inclined plateau in mid rapidity region when multiplicities more reduced produce maximum near each source centre.

We note also that if this data at $\sqrt{s}=7-8 \mathrm{Tev}$ is confirmed, it could help the interpretation of cosmic ray anomalies detected in the "knee" region above $2 \mathrm{TeV}$. The gamma ray families above $5 \mathrm{TeV}$ (near $10 \mathrm{PeV}$ in laboratory frame) exhibit steeper integral energy spectra than at lower energies. Such signal could be in relation with an important part of energy lost in the leading cluster. Those measurements in Tien Shan (EAS detectors and emulsion chambers), in Pamir or in Concorde needs developed interpretations with new models and extended simulations. The next measurements of the LHC (evaluation of total multiplicity, semi-inclusive data) extended soon up to $\sqrt{s}=14 \mathrm{TeV}$ would provide better extrapolations of the characteristics of UHE collisions favourable to a new interpretation of cosmic ray data, especially the mass composition above the knee.

The author is indebted to B. Szabelska, J. Szabelski and Z. Plebaniak for their help in developing the GHOST model and further implementation in the INTEST option of CORSIKA. This work was performed thanks to the French-Polish Collaboration Agreement IN2P3-COPIN.

\section{References}

[1] G. J. Alner et al. (UA5 Collaboration), Physics Reports 154, No 5\&6, 247-383 (1987)

[2] L. Voyvodic, AIP CONFERENCE PROCEEDINGS 276, 231-243 (1992)

[3] J. N. Capdevielle, IL NUOVO CIMENTO 19C, 5, 623-641 (1996), Rapporteur paper 24th ICRC, Rome

[4] G. J. Alner et al., UA5 collaboration, Nucl. Phys. B 291, 445, 1987

[5] G. Wolschin , Phys. Lett. B 698, 411 (2011)

[6] CMS and TOTEM collaborations, arXiv:1405. 07221, [Hep-Ex] (2014)

[7] G. J. Alner et al., UA5 collaboration, ZPC 33, 1 (1986)

[8] R. Aaij et al., LHCb collaboration, Eur. Phys. J C 72, No 4, 1947 (2012)

[9] J. N. Capdevielle et al., J. Phys. G 36, 075205 (2009)

[10] D. Heck, J. Knapp, J.N. Capdevielle, G. Schatz and T. Thouw, FZK A report-6019 ed. FZK The CORSIKA Air Shower Simulation Program, Karlsruhe (1998)

[11] S. I. Nikolski Nuclear Physics B 39A, 228 (1988) 